\title{
On a Theorem of Gauss
}

By Hansraj Gupta, Hoshiarpur (Punjab).

(Received 3rd August, 1934; received in revised form 26 th November, 1934.

Read 2nd November, 1934.)

\section{$\S 1$. Introduction.}

Professor Hemraj ${ }^{1}$ has given a proof of a part of a theorem of Gauss without using the theory of quadratic residues. Proceeding on similar lines, I have obtained a complete proof which is rather simpler and certainly more concise.

In what follows $G(n, r)$ denotes $^{2}$ the sum of the products of the first $n$ natural numbers taken $r$ at a time; $\{n, m\}$ denotes as usual the greatest common factor of the two non-zero positive integers $n$ and $m ; p$ stands for an odd prime unless stated otherwise; and $a, b, m, n$, $i, j, k, \mu, r$, etc., stand for positive integers or zero.

I write $a<. n$ when $\{a, n\}=1$ and $a<n$; and denote by $\Pi(a<. n)$ the product of all $a$ 's less than $n$ and prime to it.

If $n \equiv 0 \quad\left(\bmod p^{\mu}\right)$, but $\neq 0 \quad\left(\bmod p^{\mu+1}\right), p \geqq 2$, then I say that $n$ is $\mu$-potent in $p$, or that the $p$-potency of $n$ is $\mu$. We have $\mu=0$ when $n \neq 0(\bmod p)$.

In my proof of Gauss' Theorem, viz.

$$
\begin{aligned}
\Pi(a<. m) & \equiv-1 \quad(\bmod m) \text { when } m=2^{2}, p^{\mu}, 2 p^{\mu}, \\
& \equiv 1 \quad(\bmod m) \text { otherwise, }
\end{aligned}
$$

I make use of the lemmas of $\S 2$.

§2. LemMa 1. If a be the p-potency of $r$, then the p-potency of $\left(\begin{array}{c}p^{\mu} \\ r\end{array}\right)$ is $\mu-a$, where $1 \leqq r \leqq p^{\mu}$ and $p \geqq 2$.

We have $\left(\begin{array}{c}p^{\mu} \\ r\end{array}\right)=\frac{p^{\mu} !}{r !\left(p^{\mu}-r\right) !}$.

Therefore the $p$-potency of $\left(\begin{array}{c}p^{\mu} \\ r\end{array}\right)$

$$
=\sum_{\kappa=1}^{\mu}\left\{\left[\frac{p^{\mu}}{p^{\kappa}}\right]-\left[\frac{r}{p^{\kappa}}\right]-\left[\frac{p^{\mu}-r}{p^{\kappa}}\right]\right\}=\sum_{\kappa=1}^{\mu} \lambda_{\kappa}
$$

where $\lambda_{\kappa}=0$ or 1 according as $r \equiv 0$ or $\neq 0\left(\bmod p^{\kappa}\right)$. Since $r \equiv 0\left(\bmod p^{a}\right)$ but $\neq 0\left(\bmod p^{a+1}\right)$, it follows that the $p$-potency of $\left(\begin{array}{c}p^{\mu} \\ r\end{array}\right)$ is $\mu-a$. 
Lemma 2. The p-potency of $G\left(p^{\mu}-1, r\right)$ is greater than or equal to $\mu-\beta$, where $1 \leqq r \leqq p^{\mu}-1, p^{\beta} \leqq 2 r<p^{\beta+1}, p$ is an odd prime or 2 , and $\beta \geqq 0$.

We have ${ }^{3}$

$$
G\left(p^{\mu}-1, r\right)=\sum_{i=1}^{r}\left\{f_{i}(r)\left(\begin{array}{c}
p^{\mu} \\
2 r-i+1
\end{array}\right)\right\},
$$

where the f's are positive integers. The result stated follows immediately from Lemma 1.

Lemma 3. If $\{m, n\}=1$, then

$$
\Pi(a<. m n) \equiv\{\Pi(b<. n)\}^{\phi(m)}(\bmod n),
$$

where $\phi(m)$ denotes as usual the number of integers less than and prime to $m$.

If $b<. n$, then in the series of $m$ terms

$$
b, b+n, b+2 n, b+3 n, \ldots ., b+(m-1) n,
$$

there are $\phi(m)$ integers less than and prime to $m n$. Each of these integers $\equiv b(\bmod n)$, so that their product $\equiv\{b\}^{\phi(m)}(\bmod n)$. Giving to $b$ all values $<. n$, we get the result stated.

§3. Proof of Gauss' Theorem.

(i) We first consider the case when $m=2^{\mu}, \mu \geqq 1$. We have

$$
\begin{aligned}
& \Pi(a<.2) \equiv \pm 1 \quad(\bmod 2), \\
& \Pi\left(a<.2^{2}\right) \equiv-1 \quad\left(\bmod 2^{2}\right), \\
& \Pi\left(a<.2^{3}\right) \equiv 1.3 .5 .7 \equiv 1 \quad\left(\bmod 2^{3}\right), \\
& \Pi\left(a<.2^{4}\right) \equiv 1.3 .5 \ldots 15 \equiv 1 \quad\left(\bmod 2^{4}\right) .
\end{aligned}
$$

Suppose that $\Pi\left(a<.2^{\mu}\right) \equiv 1 \quad\left(\bmod 2^{\mu}\right)$ when $3 \leqq \mu \leqq i-1$. Then $\left.\Pi\left(a<.2^{i}\right) \equiv 1.3 .5 .7 \ldots\left(2^{i-1}-1\right) .\left(2^{i}-1\right)\left(2^{i}-3\right) \ldots .2^{i}-\left(2^{i-1}-1\right)\right\}$.

$$
\begin{aligned}
& \equiv\left\{\Pi\left(a<.2^{i-1}\right)\right\}^{2} \quad\left(\bmod 2^{i}\right) \\
& \equiv 1 \quad\left(\bmod 2^{i}\right), \text { since } 2 i-2>i .
\end{aligned}
$$

Hence by induction for $\mu \geqq 3$,

$$
\Pi\left(a<.2^{\mu}\right) \equiv 1 \quad\left(\bmod 2^{\mu}\right) .
$$

(ii) Now consider the case when $m=p^{\mu}, \mu \geqq 1$. Let $a$ be any number $<. p$, and let $\rho=p^{\mu-1}-1$. Then

$$
\prod_{\kappa=0}^{\rho}(a+\kappa p)=a^{\rho+1}+\sum_{r=1}^{\rho}\left\{G\left(p^{\mu-1}-1, r\right) p^{\gamma} a^{\rho-r+1}\right\} .
$$


Since the $p$-potency of $G\left(p^{\mu-1}-1, r\right) \cdot p^{r}$ is greater than or equal to $\mu+r-\beta-1$, where $p^{\beta} \leqq 2 r<p^{\beta+1}$, that is, greater than or equal to $\mu$, we have

$$
\prod_{\kappa=0}^{p}(a+\kappa p) \equiv a^{p+1} \quad\left(\bmod p^{\mu}\right) .
$$

Hence $\quad \Pi\left(a<\cdot p^{\mu}\right) \equiv\{\Pi(a<\cdot p)\}^{\rho+1} \equiv\{(p-1) !\}^{\rho+1} \quad\left(\bmod p^{\mu}\right)$

$$
\equiv\{j p-1\}^{+1} \quad\left(\bmod p^{\mu}\right)
$$

since $^{3}(p-1) ! \equiv-1 \quad(\bmod p) . \quad$ So by Lemma 1

$$
\Pi\left(a<\cdot p^{\mu}\right) \equiv-1 \quad\left(\bmod p^{\mu}\right) .
$$

(iii) When $m=2 p^{\mu}$, we have from Lemma 3 ,

$$
\begin{aligned}
\Pi\left(a<.2 p^{\mu}\right) & \equiv\left\{\Pi\left(a<\cdot p^{\mu}\right)\right\}^{\phi(2)} \quad\left(\bmod p^{\mu}\right) \\
& \equiv-1 \quad\left(\bmod p^{\mu}\right) .
\end{aligned}
$$

Also

$$
\begin{aligned}
\Pi\left(a<.2 p^{\mu}\right) & \equiv\{\Pi(a<.2)\}^{\phi\left(p^{\mu}\right)} \quad(\bmod 2) . \\
& \equiv 1 \equiv-1 \quad(\bmod 2) .
\end{aligned}
$$

Hence $\quad \Pi\left(a<.2 p^{\mu}\right) \equiv-1 \quad\left(\bmod 2 p^{\mu}\right)$.

(iv) When $m$ is of any form other than those already considered, Gauss' Theorem follows immediately from Lemma 3.

Let $m=p^{\mu} n$, where $\mu \geqq 1, p \geqq 2,\{n, p\}=1$, and $n>2$. Then

$$
\begin{aligned}
\Pi(a<. m) & \equiv\left\{\Pi\left(a<\cdot p^{\mu}\right)\right\}^{\phi(n)} \quad\left(\bmod p^{\mu}\right) \\
& \equiv 1 \quad\left(\bmod p^{\mu}\right),
\end{aligned}
$$

since $\phi(n)$ is even. Considering in this manner all the different primes present in $m$, we obtain

$\Pi(a<. m) \equiv 1 \quad(\bmod m), \quad m \neq 2^{2}, p^{\mu}, 2 p^{\mu}$, where $p \geqq 3$.

This proves Gauss' Theorem completely.

\section{REFERENCES.}

1. Hemraj, Journal Indian Math. Soc., 19 (1931), 34-39.

2. Hansraj Gupta, Journal Indian Math. Soc., 19 (1931), 1-6.

3. Hansraj Gupta, Proc. Edinburgh Math. Soc., 4 (1934-35), 61, equ. (1.3).

Note ADDED IN PROOF. For completion of the proof discussed in reference 1 above, see Hemraj, Mathematics Student, 2 (1934), 140-148. 